\title{
Towards the experimental clarification of quarkonium polarization
}

\author{
Pietro Faccioli $^{1}$, Carlos Lourenço $^{2, \text { a }}$, João Seixas ${ }^{1,3}$, Hermine K. Wöhri $^{1}$ \\ ${ }^{1}$ Laboratório de Instrumentação e Física Experimental de Partículas (LIP), Lisbon, Portugal \\ ${ }^{2}$ CERN, Geneva, Switzerland \\ ${ }^{3}$ Physics Department, Instituto Superior Técnico (IST), Lisbon, Portugal
}

Received: 13 June 2010 / Revised: 3 August 2010 / Published online: 1 September 2010

(C) The Author(s) 2010. This article is published with open access at Springerlink.com

\begin{abstract}
We highlight issues which are often underestimated in the experimental analyses on quarkonium polarization: the relation between the parameters of the angular distributions and the angular momentum composition of the quarkonium, the importance of the choice of the reference frame, the interplay between observed decay and production kinematics, and the consequent influence of the experimental acceptance on the comparison between experimental measurements and theoretical calculations. Given the puzzles raised by the available experimental results, new measurements must provide more detailed information, such that physical conclusions can be derived without relying on model-dependent assumptions. We describe a frame-invariant formalism which minimizes the dependence of the measurements on the experimental acceptance, facilitates the comparison with theoretical calculations, and probes systematic effects due to experimental biases. This formalism is a direct and generic consequence of the rotational invariance of the dilepton decay distribution and is independent of any assumptions specific to particular models of quarkonium production. The use of this improved approach, which exploits the intrinsic multidimensionality of the problem, will significantly contribute to a faster progress in our understanding of quarkonium production, especially if adopted as a common analysis framework by the LHC experiments, which will soon perform analyses of quarkonium polarization in proton-proton collisions.
\end{abstract}

\section{Introduction}

Detailed studies of quarkonium production should provide significant progress in our understanding of quantum chromodynamics (QCD) [1]. However, our present understand-

a e-mail: carlos.lourenco@cern.ch ing of this physics topic is rather limited, despite the multitude of experimental data accumulated over more than 30 years. The $p_{\mathrm{T}}$ differential $\mathrm{J} / \psi$ and $\psi^{\prime}$ direct production cross sections measured (in the mid 1990's) by CDF, in $\mathrm{p} \overline{\mathrm{p}}$ collisions at $1.8 \mathrm{TeV}$ [2], were seen to be around 50 times larger than the available expectations, based on leading order calculations made in the scope of the Colour Singlet Model. The non-relativistic QCD (NRQCD) framework $[3,4]$, where quarkonia can also be produced as coloured quark pairs, succeeded in describing the measurements, opening a new chapter in the studies of quarkonium production physics. However, these calculations depend on non-perturbative parameters, the long distance colour octet matrix elements, which have been freely adjusted to the data, thereby decreasing the impact of the resulting agreement between data and calculations. More recently, calculations of next-to-leading-order (NLO) QCD corrections to colour-singlet quarkonium production showed an important increase of the high- $p_{\mathrm{T}}$ rate, significantly decreasing the colour-octet component needed to reproduce the quarkonium production cross sections measured at the Tevatron [5].

Given this situation, differential cross sections are clearly insufficient information to ensure further progress in our understanding of quarkonium production. Experimental studies of the polarization of the $J^{\mathrm{PC}}=1^{--}$quarkonium states, which decay into lepton pairs, will certainly provide very useful complementary information. In fact, the competing mechanisms dominating in the different theoretical approaches lead to very different expected polarizations of the produced quarkonia. On one hand, the NRQCD calculations [6-8], dominated by the colour-octet component, predict that, at Tevatron or LHC energies and at asymptotically high $p_{\mathrm{T}}$, the directly produced $\psi^{\prime}$ and $\mathrm{J} / \psi$ mesons are produced almost fully transversely polarized (i.e. with dominant angular momentum component $J_{z}= \pm 1$ ) with respect to their own momentum direction (the helicity frame). 
On the other hand, according to the new NLO calculations of colour-singlet quarkonium production [5] these states should show a strong longitudinal $\left(J_{z}=0\right)$ polarization component.

Having two very different theoretical predictions appears to be an ideal situation when seen from an experimentalist's perspective, as one may think that it should be relatively straightforward to discriminate between the two theory frameworks using experimental measurements. Somewhat surprisingly, however, this is not the case. In fact, the present experimental knowledge is incomplete and contradictory. Studies of the $\psi^{\prime}$ polarization have been published on the basis of data collected by the CDF II experiment [9]. Unfortunately, the large experimental uncertainties caused by the small size of the data samples prevent from drawing meaningful conclusions. In principle, a more precise test of the theoretical predictions should be provided by the $\mathrm{J} / \psi$ data, given their much higher statistical accuracy. However, the experimental perspective is more complicated in this case, because a significant fraction (around one third [10]) of promptly produced $\mathrm{J} / \psi$ mesons (i.e. excluding contributions from B hadron decays) comes from $\chi_{c}$ and $\psi^{\prime}$ feeddown decays. This sizeable source of indirectly produced $\mathrm{J} / \psi$ mesons is not subtracted from the current measurements, and its kinematic dependence is not precisely known. Despite this limitation, it seems safe to say that the pattern measured by CDF [9] of a slightly longitudinal polarization of the inclusive prompt $\mathrm{J} / \psi$ 's is incompatible with any of the two theory approaches mentioned above. The situation is further complicated by the intriguing lack of continuity between fixed-target and collider results, which can only be interpreted in the framework of some specific (and speculative) assumptions still to be tested [11].

The $b \bar{b}$ system should satisfy the non-relativistic approximation much better than the $c \bar{c}$ case. For this reason, the $\Upsilon$ data are expected to represent the most decisive test of NRQCD. However, the comparison with the existing $\Upsilon(1 S)$ polarization data from the Tevatron $[12,13]$ is far from conclusive. The results indicate that, for $p_{\mathrm{T}}<15 \mathrm{GeV} / c$, the $\Upsilon(1 S)$ is produced either unpolarized (CDF) or longitudinally polarized (D0) in the helicity frame, and this discrepancy cannot be reasonably attributed to the different rapidity windows covered by the two experiments. Furthermore, the precision of the data for $p_{\mathrm{T}}$ higher than $15 \mathrm{GeV} / c$ is not sufficient to provide a significant test of the crucial hypothesis that very high- $p_{\mathrm{T}}$ quarkonia, produced by the fragmentation of an outgoing (almost on-shell) gluon, are fully transversely polarized along their own direction. At lower energy and $p_{\mathrm{T}}$, the E866 experiment [14] has shown yet a different polarization pattern: the $\Upsilon(2 S)$ and $\Upsilon(3 S)$ states have maximal transverse polarization, with no significant dependence on transverse or longitudinal momentum, with respect to the direction of motion of the colliding hadrons (Collins-Soper frame). Unexpectedly, the $\Upsilon(1 S)$, whose spin and angular momentum properties are identical to the ones of the heavier $\Upsilon$ states, is, instead, found to be only weakly polarized. These results give interesting physical indications. First, the maximal polarization of $\Upsilon(2 S)$ and $\Upsilon(3 S)$ along the direction of the interacting particles places strong constraints on the topology and spin properties of the underlying elementary production process. Second, the small $\Upsilon(1 S)$ polarization suggests that the bottomonium family may have a peculiar feed-down hierarchy, with a very significant fraction of the lower mass state being produced indirectly; at the same time, the polarization of the $\Upsilon$ 's coming from $\chi_{b}$ decays should be substantially different from the polarization of the directly produced ones.

This rather confusing situation demands a significant improvement in the accuracy and detail of the polarization measurements, ideally distinguishing between the properties of directly and indirectly produced states. The lack of a consistent description of the polarization properties represents today's biggest uncertainty in the simulation of the LHC quarkonium production measurements and will probably be the largest contribution to the systematic error affecting the measurements of quarkonium production cross sections and kinematic distributions. Indeed, the probability that the detector accepts lepton pairs resulting from decays of quarkonium states is strongly dependent on the polarization of those states. Therefore, even from a purely experimental point of view it is very unsatisfactory that essential properties of these objects, such as kinematic details of how they decay into lepton pairs (on which their reconstruction is based), are subjected to such a high degree of uncertainty.

It is true that measurements of the quarkonium decay angular distributions are challenging, multi-dimensional kinematic problems, which require large event samples and a very high level of accuracy in the subtraction of spurious kinematic correlations induced by the detector acceptance. The complexity of the experimental problems which have to be faced in the polarization measurements is testified, for example, by the disagreement between the CDF results obtained in Run I and Run II for the $\mathrm{J} / \psi[9,15]$ and by the contradictory results obtained by $\mathrm{CDF}$ and $\mathrm{D} 0$ for the $\Upsilon(1 S)$. However, it is also true, as we shall emphasize in this paper, that most experiments have exploited, and presented in the published reports, only a fraction of the physical information derivable from the data. This happens, for example, when the measurement is performed in only one polarization frame and is limited to the polar projection of the decay angular distribution. As we have already argued in [11], these incomplete measurements do not allow definite physical conclusions. At best, they confine such conclusions to a genuinely model-dependent framework. Moreover, such a fragmentary description of the observed physical process obviously reduces the chances of detecting possible biases induced by not fully controlled systematic effects. 
In this paper we review the mathematical framework for the description of the observable polarization of quarkonium states decaying electromagnetically into lepton pairs. We focus our attention on aspects that need to be taken in consideration in the analysis of the data, so as to maximize the physical significance of the measurement and provide all elements for its unambiguous interpretation within any theoretical framework. By increasing the level of detail of the physical information extracted from the data, the proposed methodologies also offer the possibility of performing consistency checks which can expose unaccounted detector or analysis biases. The only relevant theoretical ingredients of our discussion are the quantum properties of angular momentum and basic conservation rules of the electromagnetic interaction (parity, fermion chirality). All the results presented here are, therefore, valid in general for any quarkonium production mechanism.

In Sect. 2 we define the concept of polarization and give simple examples of how basic production mechanisms can lead to the formation of polarized quarkonium states. We then focus on the dilepton decay distribution of ${ }^{3} S_{1}$ quarkonia, a relatively simple case, and provide detailed geometric and kinematic considerations. In Sect. 3 we recall the basic principles leading to the general expression describing the angular distribution of the decay products, while in Sect. 4 we describe how the observed anisotropy parameters depend on the choice of the reference frame. Section 5 is devoted to a detailed description of how the production kinematics influences the observed polarization, depending on the quarkonium momentum and on the observation frame. We also discuss quantitatively the influence of the intrinsic parton transverse momenta on the polarization measurement when the natural axis is along the relative flight direction of the colliding partons. In Sect. 6 we derive the existence of a frame-independent identity which relates the observable parameters of the decay distribution to one frame-invariant polarization parameter. We discuss how this relation, formally including the Lam-Tung identity [16] as a particular case, improves the representation of polarization results and can be used to perform consistency checks in the experimental analyses. We continue with some remarks, given in Sect. 7, on how the existence of intrinsic parton transverse momentum affects the polarization measurement. We conclude, in Sect. 8, with a few examples inspired from existing experimental measurements, which should provide concrete evidence for the usefulness of the approaches discussed in this paper, in view of ensuring an improved understanding of quarkonium production.

\section{Basic polarization concepts}

Because of angular momentum conservation and basic symmetries of the electromagnetic and strong interactions, a particle produced in a certain superposition of elementary mechanisms may be observed preferentially in a state belonging to a definite subset of the possible eigenstates of the angular momentum component $J_{z}$ along a characteristic quantization axis. When this happens, the particle is said to be polarized. Figure 1 shows examples of leading-order diagrams of elementary production processes giving rise to different types of polarizations.

Vector $\left(J=1^{--}\right)$quarkonia have the same charge-parity as an electron-positron pair and can be produced in electronpositron annihilation, via an intermediate photon (Fig. 1(a)). The states originating from this process are polarized, as a consequence of helicity conservation, a general property of QED in the relativistic (massless) limit. The dynamics of the coupling of electrons to photons is, in fact, described by terms of the form $\bar{u} \gamma^{\mu} u=\bar{u}_{\mathrm{L}} \gamma^{\mu} u_{\mathrm{L}}+\bar{u}_{\mathrm{R}} \gamma^{\mu} u_{\mathrm{R}}$, where $\gamma^{\mu}$ are the Dirac matrices, $u$ is the electron spinor, and $\mathrm{L}(\mathrm{R})$ indicate its left-handed (right-handed) chiral components. Terms with opposite chiral components are absent, meaning that the fermion chirality is preserved in the interaction with a photon. When the fermions are assumed to have zero mass, so that the direction of their momenta cannot be reversed by any Lorentz transformation, left-handed and righthanded chiral components become eigenstates of the helicity operator $h=\boldsymbol{S} \cdot \boldsymbol{p} /|\boldsymbol{p}|$, corresponding to the projection of the spin on the momentum direction. In this case, chirality conservation becomes helicity conservation. In the diagram of Fig. 1(a), this rule implies that the annihilating electron and positron must have opposite helicities, because the intermediate photon has zero (fermion) helicity. Since in the laboratory their momenta are opposite, their spins must be a)

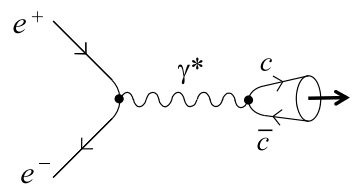

b)

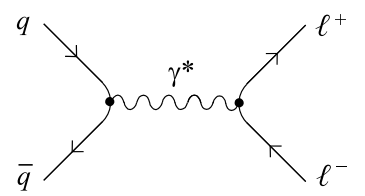

c)

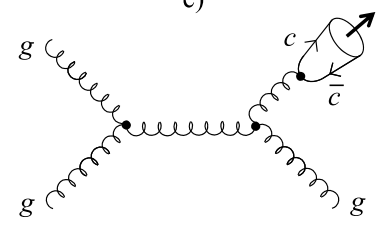

Fig. 1 Examples of leading-order diagrams for production mechanisms giving rise to observable polarizations: (a) vector quarkonium production in electron-positron annihilation; (b) Drell-Yan production in quark-antiquark annihilation; (c) quarkonium production by gluon fragmentation to colour-octet $c \bar{c}$ 
parallel. Because of angular momentum conservation, the produced quarkonium has, thus, angular momentum component $J_{z}= \pm 1$ along the direction of the colliding leptons. This precise QED prediction (the relative amplitude for the $J_{z}=0$ component is of order $m_{e} / E_{e} \simeq 3 \times 10^{-4}$ for $\mathrm{J} / \psi$ production and smaller for $\Upsilon$ production) is commonly used as a base assumption in quarkonium measurements in electron-positron annihilations (as, for example, in the recent analysis of [17]). The fact that the dilepton system coupled to a photon is a pure $J_{z}= \pm 1$ state is also an essential ingredient in the determination of the expression for the dilepton-decay angular distributions of vector quarkonia (see Sect. 3).

The same reasoning can be applied to the production of Drell-Yan lepton pairs in quark-antiquark annihilation (Fig. 1(b)): the quark and antiquark, in the limit of vanishing masses, must annihilate with opposite helicities, resulting in a dilepton state having $J_{z}= \pm 1$ along the direction of their relative velocity. The experimental verification of this basic mechanism has reached an impressive level of accuracy [14]. Quark helicity is conserved also in QCD, when the masses can be neglected. Similarly to the DrellYan case, quarkonia originating from quark-antiquark annihilation (into intermediate gluons) will thus tend, provided they are produced alone, to have their angular momentum vectors "aligned" $\left(J_{z}= \pm 1\right)$ along the beam direction. This prediction is in good agreement with the $\chi_{c 1}, \chi_{c 2}$ and $\psi^{\prime}$ polarizations measured in low-energy proton-antiproton collisions [18-20].

At very high $p_{\mathrm{T}}$, quarkonium production at hadron colliders should mainly proceed by gluon fragmentation [21]. In NRQCD, heavy-quark velocity scaling rules for the nonperturbative matrix elements, combined with the $\alpha_{S}$ and $1 / p_{\text {T }}$ power counting rules for the parton cross sections, predict that $\mathrm{J} / \psi$ and $\psi^{\prime}$ production at high $p_{\mathrm{T}}$ is dominated by gluon fragmentation into the colour-octet state $c \bar{c}\left[{ }^{3} S_{1}^{(8)}\right]$ (Fig. 1(c)). Transitions of the gluon to other allowed colour and angular momentum configurations, containing the $c \bar{c}$ in either a colour-singlet or a colour-octet state, with spin $S=0,1$ and angular momentum $L=0,1,2, \ldots$, as well as additional gluons $\left(c \bar{c}\left[{ }^{1} S_{0}^{(8)}\right] g, c \bar{c}\left[{ }^{3} P_{J}^{(8)}\right] g, c \bar{c}\left[{ }^{3} S_{1}^{(1)}\right] g g\right.$, etc.), are more and more suppressed with increasing $p_{\mathrm{T}}$. Up to small corrections, the fragmenting gluon is believed to be on shell and have, therefore, helicity \pm 1 . This property is inherited by the $c \bar{c}\left[{ }^{3} S_{1}^{(8)}\right]$ state and remains intact during the non-perturbative transition to the colour-neutral physical state, via soft-gluon emission. In this model, the observed charmonium has, thus, angular momentum component $J_{z}= \pm 1$, this time not along the direction of the beam, but along its own flight direction.

"Unpolarized" quarkonium has the same probability, $1 /(2 J+1)$, to be found in each of the angular momentum eigenstates, $J_{z}=-J,-J+1, \ldots,+J$. This is the case, for example, in the colour evaporation model [22, 23]. In this framework, similarly to NRQCD, the $Q \bar{Q}$ pair is produced at short distances in any colour and angular momentum configuration. However, contrary to NRQCD, no hierarchy constraints are imposed on these configurations, so that the cross section turns out to be dominated by $Q \bar{Q}$ pairs with vanishing angular momentum $\left({ }^{1} S_{0}\right)$, in either coloursinglet or colour-octet states. In their long distance evolution through soft gluon emissions, $J=0$ states get their colour randomized, assuming the correct quantum numbers of the physical quarkonium. As a result, the final angular momentum vector $\boldsymbol{J}$ has no preferred alignment.

In two-body decays (such as the ${ }^{3} S_{1} \rightarrow \ell^{+} \ell^{-}$case considered in this paper), the geometrical shape of the angular distribution of the two decay products (emitted back-toback in the quarkonium rest frame) reflects the polarization of the quarkonium state. A spherically symmetric distribution would mean that the quarkonium would be, on average, unpolarized. Anisotropic distributions signal polarized production.

The measurement of the distribution requires the choice of a coordinate system, with respect to which the momentum of one of the two decay products is expressed in spherical coordinates. In inclusive quarkonium measurements, the axes of the coordinate system are fixed with respect to the physical reference provided by the directions of the two colliding beams as seen from the quarkonium rest frame. Figure 2 illustrates the definitions of the polar angle $\vartheta$, determined by the direction of one of the two decay products (e.g. the positive lepton) with respect to the chosen polar axis, and of the azimuthal angle $\varphi$, measured with respect to the plane containing the momenta of the colliding beams ("production plane"). The actual definition of the decay reference frame with respect to the beam directions is not unique. Measurements of the quarkonium decay distributions have used three different conventions for the orientation of the polar axis (see Fig. 3): the direction of the momentum of one of the two

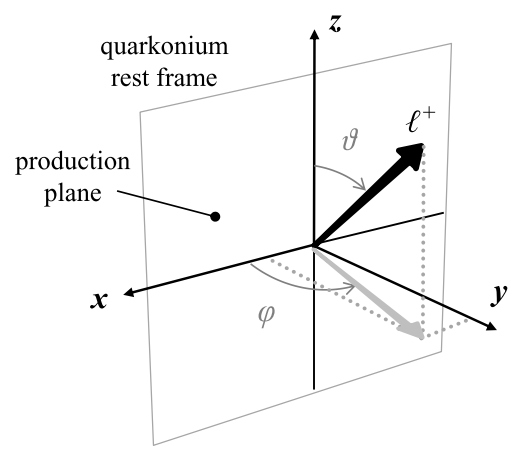

Fig. 2 The coordinate system for the measurement of a two-body decay angular distribution in the quarkonium rest frame. The $y$ axis is perpendicular to the plane containing the momenta of the colliding beams. The polarization axis $z$ is chosen according to one of the possible conventions described in Fig. 3 
Fig. 3 Illustration of three different definitions of the polarization axis $z(\mathrm{CS}$ : Collins-Soper, GJ: Gottfried-Jackson, HX: helicity) with respect to the directions of motion of the colliding beams $\left(b_{1}, b_{2}\right)$ and of the quarkonium $(\mathcal{Q})$
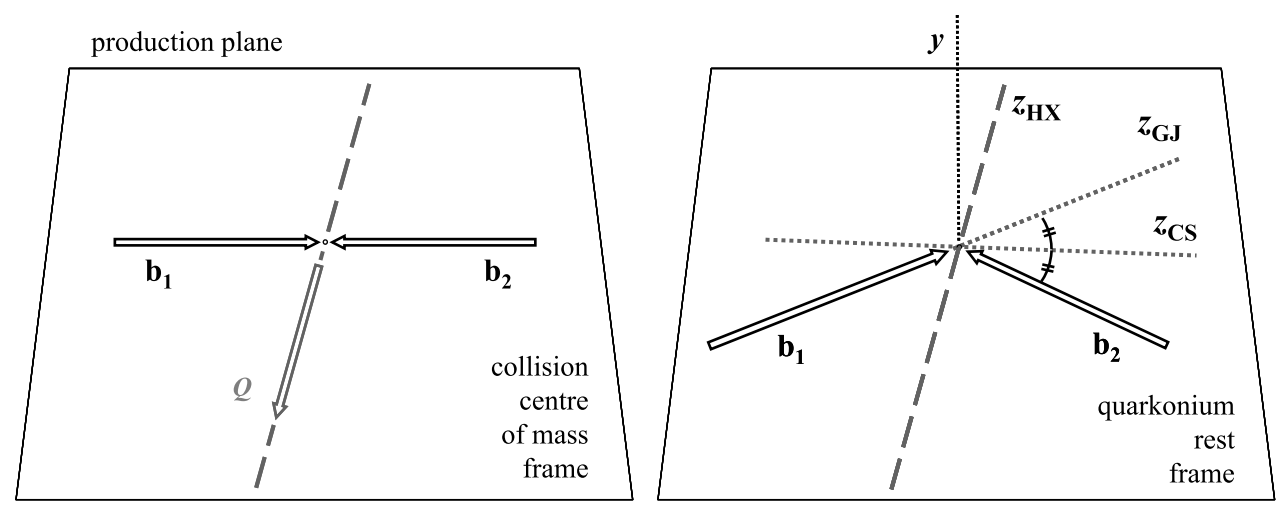

colliding beams (Gottfried-Jackson frame [24], GJ), the opposite of the direction of motion of the interaction point (i.e. the flight direction of the quarkonium itself in the centre-ofmass of the colliding beams: centre-of-mass helicity frame, HX) and the bisector of the angle between one beam and the opposite of the other beam (Collins-Soper frame [25], CS). The motivation of this latter definition is that, in hadronic collisions, it coincides with the direction of the relative motion of the colliding partons, when their transverse momenta are neglected (the validity and limits of this approximation are discussed in detail in Sect. 7). For our considerations, we will take the HX and CS frames as two extreme (physically relevant) cases, given that the GJ polar axis represents an intermediate situation. We note that these two frames differ by a rotation of $90^{\circ}$ around the $y$ axis when the quarkonium is produced at high $p_{\mathrm{T}}$ and negligible longitudinal momentum $\left(p_{\mathrm{T}} \gg\left|p_{\mathrm{L}}\right|\right)$. All definitions become coincident in the limit of zero quarkonium $p_{\mathrm{T}}$. In this limit, moreover, for symmetry reasons any azimuthal dependence of the decay distribution is physically forbidden.

We conclude this section by defining the somewhat misleading nomenclature which is commonly used (and adopted, for convenience, also in this paper) for the polarization of vector mesons. These particles share the quantum numbers of the photon and are therefore said, by analogy with the photon, to be "transversely" polarized when they have spin projection $J_{z}= \pm 1$. The counterintuitive adjective originally refers to the fact that the electromagnetic field carried by the photon oscillates in the transverse plane with respect to the photon momentum, while the photon spin is aligned along the momentum. "Longitudinal" polarization means $J_{z}=0$. By further extension, the same terms are also used to describe the "spin alignment" of vector quarkonia not only with respect to their own momenta (HX frame), but also with respect to any other chosen reference direction (such as the GJ or CS axes).

\section{Dilepton decay angular distribution}

Vector quarkonia, such as the $\mathrm{J} / \psi, \psi^{\prime}$ and $\Upsilon(n S)$ states, can decay electromagnetically into two leptons. The reconstruction of this channel represents the cleanest way, both from the experimental and theoretical perspectives, of measuring their production yields and polarizations. In this and the following sections we discuss how to determine experimentally the "spin alignment" of a vector quarkonium by measuring the dilepton decay angular distribution. For convenience we mention explicitly the $\mathrm{J} / \psi$ as the decaying particle, but considerations and results are valid for any $J=1^{--}$state.

We begin by studying the case of a single production "subprocess", here defined as a process where the $\mathrm{J} / \psi$ is formed as a given superposition of the three $J=1$ eigenstates, $J_{z}=+1,-1,0$ with respect to the polarization axis $z$ :

$|V\rangle=b_{+1}|+1\rangle+b_{-1}|-1\rangle+b_{0}|0\rangle$.

The calculations are performed in the $\mathrm{J} / \psi$ rest frame, where the common direction of the two leptons define the reference axis $z^{\prime}$, oriented conventionally along the direction of the positive lepton. The adopted notations for axes, angles and angular momentum states are illustrated in Fig. 4. Because of helicity conservation for (massless) fermions in

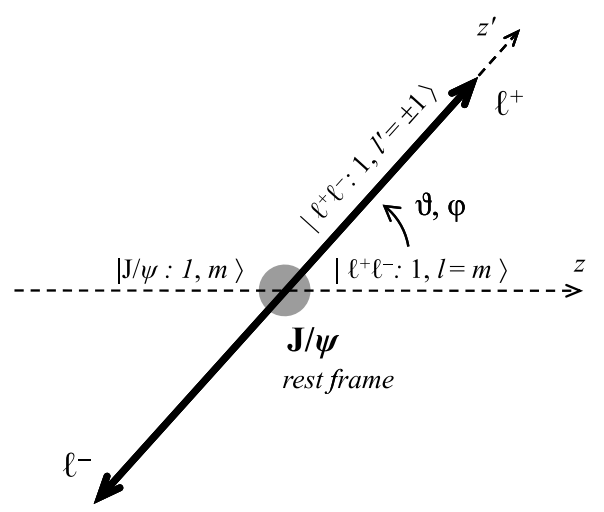

Fig. 4 Sketch of the decay $\mathrm{J} / \psi \rightarrow \ell^{+} \ell^{-}$, showing the notations used in the text for axes, angles and angular momentum states 
QED, the dilepton system coupled to a photon in the process $\mathrm{J} / \psi \rightarrow \gamma^{*} \rightarrow \ell^{+} \ell^{-}$has angular momentum projection \pm 1 along $z^{\prime}$, i.e. it can be represented as an eigenstate of $J_{z^{\prime}}$, $\left|\ell^{+} \ell^{-} ; 1, l^{\prime}\right\rangle$ with $l^{\prime}=+1$ or -1 . We want to express this state as a superposition of eigenstates of $J_{z},\left|\ell^{+} \ell^{-} ; 1, l\right\rangle$ with $l=0, \pm 1$. To perform this change of quantization axis, we use a general result of angular momentum theory, which we recall in the following paragraphs.

We indicate by $R(\alpha, \beta, \gamma)$ the rotation from a generic set of axes $(x, y, z)$ to the set $\left(x^{\prime}, y^{\prime}, z^{\prime}\right), \alpha, \beta$ and $\gamma$ denoting the Euler angles. Positive rotations are defined by the righthand rule. An eigenstate $\left|J, M^{\prime}\right\rangle$ of $J_{z^{\prime}}$ can then be expressed as a superposition of the eigenstates $|J, M\rangle$ of $J_{z}$ through the rotation transformation [26]

$\left|J, M^{\prime}\right\rangle=\sum_{M=-J}^{+J} \mathcal{D}_{M M^{\prime}}^{J}(R)|J, M\rangle$.

The (complex) rotation matrix elements $\mathcal{D}_{M M^{\prime}}^{J}$ are defined as

$\mathcal{D}_{M M^{\prime}}^{J}(\alpha, \beta, \gamma)=e^{-i M \alpha} d_{M M^{\prime}}^{J}(\beta) e^{-i M^{\prime} \gamma}$

in terms of the (real) reduced matrix elements

$$
\begin{aligned}
& d_{M M^{\prime}}^{J}(\beta) \\
& =\sum_{t=\max \left(0, M-M^{\prime}\right)}^{\min \left(J+M, J-M^{\prime}\right)}(-1)^{t} \\
& \quad \times \frac{\sqrt{(J+M) !(J-M) !\left(J+M^{\prime}\right) !\left(J-M^{\prime}\right) !}}{(J+M-t) !\left(J-M^{\prime}-t\right) ! t !\left(t-M+M^{\prime}\right) !} \\
& \quad \times\left(\cos \frac{\beta}{2}\right)^{2 J+M-M^{\prime}-2 t}\left(\sin \frac{\beta}{2}\right)^{2 t-M+M^{\prime}} .
\end{aligned}
$$

The rotation we need in our case has the effect of bringing one quantization axis $(z)$ to coincide with another $\left(z^{\prime}\right)$. The most general rotation performing this projection can be parametrized with $\beta=\vartheta$ and $\alpha=-\gamma=\varphi$. The dilepton angular momentum state is therefore expressed in terms of eigenstates of $J_{z}$ as

$\left|\ell^{+} \ell^{-} ; 1, l^{\prime}\right\rangle=\sum_{l=0, \pm 1} \mathcal{D}_{l l^{\prime}}^{1}(\varphi, \vartheta,-\varphi)\left|\ell^{+} \ell^{-} ; 1, l\right\rangle$.

The amplitude of the partial process $\mathrm{J} / \psi(m) \rightarrow \ell^{+} \ell^{-}\left(l^{\prime}\right)$ represented in Fig. 4 is

$$
\begin{aligned}
B_{m l^{\prime}} & =\sum_{l=0, \pm 1} \mathcal{D}_{l l^{\prime}}^{1 *}(\varphi, \vartheta,-\varphi)\left\langle\ell^{+} \ell^{-} ; 1, l|\mathcal{B}| \mathrm{J} / \psi ; 1, m\right\rangle \\
& =B \mathcal{D}_{m l^{\prime}}^{1 *}(\varphi, \vartheta,-\varphi),
\end{aligned}
$$

where we imposed that the transition operator $\mathcal{B}$ is of the form $\left\langle\ell^{+} \ell^{-} ; 1, l|\mathcal{B}| \mathrm{J} / \psi ; 1, m\right\rangle=B \delta_{m l}$ because of angular momentum conservation, with $B$ independent of $m$ (for rotational invariance). The total amplitude for $\mathrm{J} / \psi \rightarrow \ell^{+} \ell^{-}\left(l^{\prime}\right)$, where the $\mathrm{J} / \psi$ is given by the superposition written in (1), is

$$
\begin{aligned}
B_{l^{\prime}} & =\sum_{m=0, \pm 1} b_{m} B \mathcal{D}_{m l^{\prime}}^{1 *}(\varphi, \vartheta,-\varphi) \\
& =\sum_{m=0, \pm 1} a_{m} \mathcal{D}_{m l^{\prime}}^{1 *}(\varphi, \vartheta,-\varphi)
\end{aligned}
$$

The probability of the transition is obtained by squaring (7) and summing over the (unobserved) spin alignments $\left(l^{\prime}= \pm 1\right)$ of the dilepton system, with equal weights attributed, for parity conservation, to the two configurations. Using (3), with $d_{0, \pm 1}^{1}= \pm \sin \vartheta / \sqrt{2}, d_{ \pm 1, \pm 1}^{1}=(1+\cos \vartheta) / 2$ and $d_{ \pm 1, \mp 1}^{1}=(1-\cos \vartheta) / 2$ (from (4)), one obtains the angular distribution

$W(\cos \vartheta, \varphi)$

$$
\begin{aligned}
\propto & \sum_{l^{\prime}= \pm 1}\left|B_{l^{\prime}}\right|^{2} \propto \frac{\mathcal{N}}{\left(3+\lambda_{\vartheta}\right)}\left(1+\lambda_{\vartheta} \cos ^{2} \vartheta\right. \\
& +\lambda_{\varphi} \sin ^{2} \vartheta \cos 2 \varphi+\lambda_{\vartheta \varphi} \sin 2 \vartheta \cos \varphi \\
& \left.+\lambda_{\varphi}^{\perp} \sin ^{2} \vartheta \sin 2 \varphi+\lambda_{\vartheta}^{\perp} \sin 2 \vartheta \sin \varphi\right),
\end{aligned}
$$

with $\mathcal{N}=\left|a_{0}\right|^{2}+\left|a_{+1}\right|^{2}+\left|a_{-1}\right|^{2}$ and

$\lambda_{\vartheta}=\frac{\mathcal{N}-3\left|a_{0}\right|^{2}}{\mathcal{N}+\left|a_{0}\right|^{2}}$,

$\lambda_{\varphi}=\frac{2 \operatorname{Re}\left[a_{+1}^{*} a_{-1}\right]}{\mathcal{N}+\left|a_{0}\right|^{2}}$,

$\lambda_{\vartheta \varphi}=\frac{\sqrt{2} \operatorname{Re}\left[a_{0}^{*}\left(a_{+1}-a_{-1}\right)\right]}{\mathcal{N}+\left|a_{0}\right|^{2}}$,

$\lambda_{\varphi}^{\perp}=\frac{2 \operatorname{Im}\left[a_{+1}^{*} a_{-1}\right]}{\mathcal{N}+\left|a_{0}\right|^{2}}$,

$\lambda_{\vartheta \varphi}^{\perp}=\frac{-\sqrt{2} \operatorname{Im}\left[a_{0}^{*}\left(a_{+1}+a_{-1}\right)\right]}{\mathcal{N}+\left|a_{0}\right|^{2}}$.

Figure 5 shows the shapes of the dilepton decay distributions in the two polarization cases $m= \pm 1$ (a) and $m=0$ (b); the $m=+1$ and $m=-1$ configurations are indistinguishable because of rotation invariance. The same distributions are also shown, in panels (c) and (d), as seen when studied in frames rotated by $90^{\circ}$, anticipating the discussion in Sect. 4 .

It is worth noticing that it is impossible to chose the decay amplitudes $a_{m}$ and, therefore, the component amplitudes $b_{m}$ such that all decay parameters in (8) vanish. This means that the angular distribution of the decay of a $J=1$ state is never intrinsically isotropic. Even if it is conceivable that a lucky superposition of different production processes 

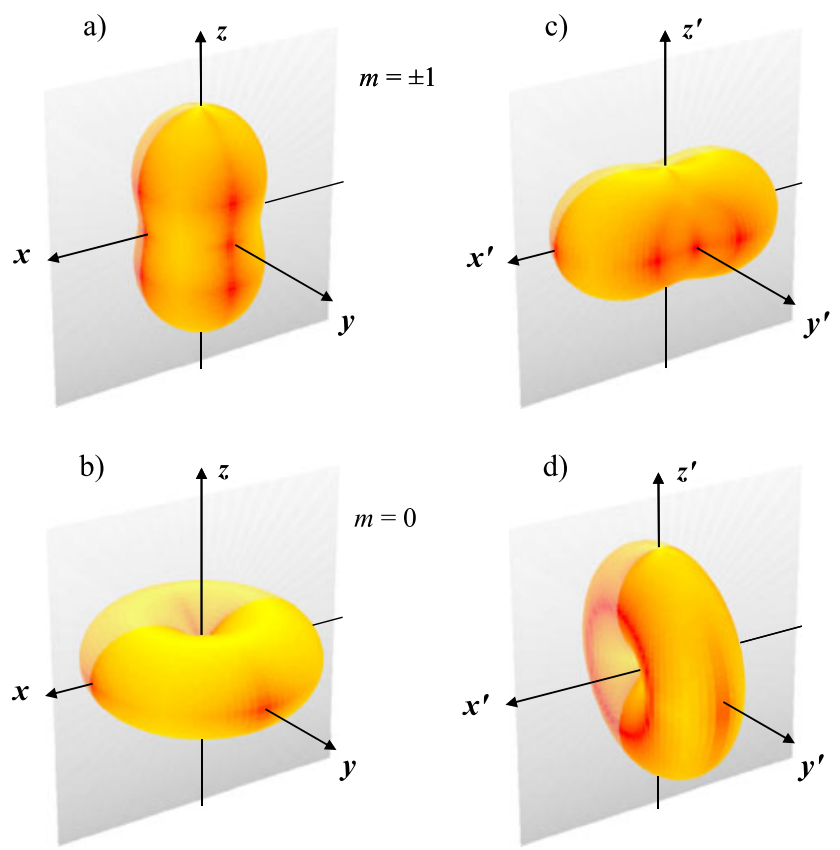

Fig. 5 Graphical representation of the dilepton decay distribution of "transversely" (a) and "longitudinally" (b) polarized quarkonium in the natural frame and in frames rotated by $90^{\circ}$ (c-d). The probability of the lepton emission in one direction is represented by the distance of the corresponding surface point from the origin

might lead to a fortuitous cancellation of all decay parameters, such an exceptional case would signal a non-trivial physical polarization scenario, caused by spin randomization effects, or (semi-)exclusive configurations in which the observed state is produced together with certain final state objects. In other words, polarization is an essential property of the quarkonium states. This remark is particularly relevant when we consider that all existing Monte Carlo generators use an isotropic dilepton distribution as the default option for quarkonium production in hadronic collisions, a non-trivial assumption with a strong influence on the acceptance estimates and, therefore, on both normalizations and kinematic dependencies of the measured quarkonium cross sections.

In this paper we only consider inclusive production. Therefore, the only possible experimental definition of the $x z$ plane coincides with the production plane, containing the directions of the colliding particles and of the decaying particle itself. The last two terms in (8) introduce an asymmetry of the distribution by reflection with respect to the production plane, an asymmetry which is not forbidden in individual (parity-conserving) events. In hadronic collisions, due to the intrinsic parton transverse momenta, for example, the "natural" polarization plane does not coincide event-byevent with the experimental production plane. However, the symmetry by reflection must be a property of the observed event distribution when only parity-conserving processes contribute. Indeed, the terms in $\sin ^{2} \vartheta \sin 2 \varphi$ and $\sin 2 \vartheta \sin \varphi$ are unobservable, because they vanish on average.

In the presence of $n$ contributing production processes with weights $f^{(i)}$, the most general observable distribution can be written as

$$
\begin{aligned}
W(\cos \vartheta, \varphi)= & \sum_{i=1}^{n} f^{(i)} W^{(i)}(\cos \vartheta, \varphi) \\
& \propto \frac{1}{\left(3+\lambda_{\vartheta}\right)}\left(1+\lambda_{\vartheta} \cos ^{2} \vartheta\right. \\
& \left.+\lambda_{\varphi} \sin ^{2} \vartheta \cos 2 \varphi+\lambda_{\vartheta \varphi} \sin 2 \vartheta \cos \varphi\right),
\end{aligned}
$$

where $W^{(i)}(\cos \vartheta, \varphi)$ is the "elementary" decay distribution corresponding to a single subprocess (given by (8) and (9), adding the index $(i)$ to the decay parameters) and each of the three observable shape parameters, $X=\lambda_{\vartheta}, \lambda_{\varphi}$ and $\lambda_{\vartheta \varphi}$, is a weighted average of the corresponding parameters, $X^{(i)}$, characterizing the single subprocesses,

$X=\sum_{i=1}^{n} \frac{f^{(i)} \mathcal{N}^{(i)}}{3+\lambda_{\vartheta}^{(i)}} X^{(i)} / \sum_{i=1}^{n} \frac{f^{(i)} \mathcal{N}^{(i)}}{3+\lambda_{\vartheta}^{(i)}}$.

We conclude this section with the derivation of formulae which can be used for the determination of the parameters of the observed angular distribution, as an alternative to a multi-parameter fit to the function in (10). The integration over either $\varphi$ or $\cos \vartheta$ leads to one-dimensional angular distributions,

$W(\cos \vartheta) \propto \frac{1}{3+\lambda_{\vartheta}}\left(1+\lambda_{\vartheta} \cos ^{2} \vartheta\right)$,

$W(\varphi) \propto 1+\frac{2 \lambda_{\varphi}}{3+\lambda_{\vartheta}} \cos 2 \varphi$,

from which $\lambda_{\vartheta}$ and $\lambda_{\varphi}$ can be determined in two separate steps, possibly improving the stability of the fit procedures in low-statistics analyses. The "diagonal" term, $\lambda_{\vartheta \varphi}$, vanishes in both integrations but can be extracted, for example, by defining the variable $\tilde{\varphi}$ as

$\tilde{\varphi}= \begin{cases}\varphi-\frac{3}{4} \pi & \text { for } \cos \vartheta<0 \\ \varphi-\frac{\pi}{4} & \text { for } \cos \vartheta>0\end{cases}$

(adding or subtracting $2 \pi$ when $\tilde{\varphi}$ does not fall into one continuous range, e.g. $[0,2 \pi]$ ) and measuring the distribution

$W(\tilde{\varphi}) \propto 1+\frac{\sqrt{2} \lambda_{\vartheta \varphi}}{3+\lambda_{\vartheta}} \cos \tilde{\varphi}$.

Each of the three parameters can also be expressed in terms of an asymmetry between the populations of two angular topologies (which are equiprobable only in the unpolarized 
case):

$$
\begin{aligned}
& \frac{P(|\cos \vartheta|>1 / 2)-P(|\cos \vartheta|<1 / 2)}{P(|\cos \vartheta|>1 / 2)+P(|\cos \vartheta|<1 / 2)}=\frac{3}{4} \frac{\lambda_{\vartheta}}{3+\lambda_{\vartheta}}, \\
& \frac{P(\cos 2 \varphi>0)-P(\cos 2 \varphi<0)}{P(\cos 2 \varphi>0)+P(\cos 2 \varphi<0)}=\frac{2}{\pi} \frac{2 \lambda_{\varphi}}{3+\lambda_{\vartheta}}, \\
& \frac{P(\sin 2 \vartheta \cos \varphi>0)-P(\sin 2 \vartheta \cos \varphi<0)}{P(\sin 2 \vartheta \cos \varphi>0)+P(\sin 2 \vartheta \cos \varphi<0)}=\frac{2}{\pi} \frac{2 \lambda_{\vartheta}}{3+\lambda_{\vartheta}} .
\end{aligned}
$$

In analyses applying efficiency corrections to the reconstructed angular spectra, the use of these formulae may require an iterative re-weighting of the Monte Carlo data, in order to compensate for the effect of the non-uniformity of those experimental corrections. In "ideal" experiments with uniform acceptance and efficiencies over $\cos \vartheta$ and $\varphi$ (such as in Monte Carlo studies at the generation level) the parameters can be obtained from the average values of certain angular distributions:

$$
\begin{aligned}
& \left\langle\cos ^{2} \vartheta\right\rangle=\frac{1+\frac{3}{5} \lambda_{\vartheta}}{3+\lambda_{\vartheta}}, \\
& \langle\cos 2 \varphi\rangle=\frac{\lambda_{\varphi}}{3+\lambda_{\vartheta}}, \\
& \langle\sin 2 \vartheta \cos \varphi\rangle=\frac{4}{5} \frac{\lambda_{\vartheta \varphi}}{3+\lambda_{\vartheta}} .
\end{aligned}
$$

\section{Dependence of the measurement on the observation frame}

All possible experimentally definable polarization axes in inclusive measurements belong to the production plane (defined in Fig. 3). We can, therefore, parametrize the transformation from an observation frame to another by one angle describing a rotation about the $y$ axis. Instead of rotating the angular momentum state vectors, we can apply a purely geometrical transformation directly to the observable angular distribution. The rotation matrix

$R_{y}(\delta)=\left(\begin{array}{ccc}\cos \delta & 0 & -\sin \delta \\ 0 & 1 & 0 \\ \sin \delta & 0 & \cos \delta\end{array}\right)$

brings the old frame to coincide with the new one, the positive sign of $\delta$ being defined by the right-hand rule (we will discuss in Sect. 5 how the sign of $\delta$ depends in an observable way on the conventions chosen for the orientation of the $z$ and $y$ axes, and how, specifically, the angle between the HX and CS axes depends on the quarkonium production kinematics). The unit vector $\hat{r}=(\sin \vartheta \cos \varphi, \sin \vartheta \sin \varphi, \cos \vartheta)$ indicating the lepton direction in the old frame is then expressed as $\hat{r}=R_{y}^{-1}(\delta) \hat{r}^{\prime}$ as a function of the coordinates in the new frame. In particular,

$\cos \vartheta=-\sin \delta \sin \vartheta^{\prime} \cos \varphi^{\prime}+\cos \delta \cos \vartheta^{\prime}$

Substituting (19) into (10), we obtain the angular distribution in the rotated frame:

$$
\begin{aligned}
& W^{\prime}\left(\cos \vartheta^{\prime}, \varphi^{\prime}\right) \\
& \propto \frac{1}{3+\lambda_{\vartheta}^{\prime}}\left(1+\lambda_{\vartheta}^{\prime} \cos ^{2} \vartheta^{\prime}\right. \\
& \left.\quad+\lambda_{\varphi}^{\prime} \sin ^{2} \vartheta^{\prime} \cos 2 \varphi^{\prime}+\lambda_{\vartheta \varphi}^{\prime} \sin 2 \vartheta^{\prime} \cos \varphi^{\prime}\right),
\end{aligned}
$$

where

$$
\begin{aligned}
& \lambda_{\vartheta}^{\prime}=\frac{\lambda_{\vartheta}-3 \Lambda}{1+\Lambda}, \quad \lambda_{\varphi}^{\prime}=\frac{\lambda_{\varphi}+\Lambda}{1+\Lambda}, \\
& \lambda_{\vartheta \varphi}^{\prime}=\frac{\lambda_{\vartheta \varphi} \cos 2 \delta-\frac{1}{2}\left(\lambda_{\vartheta}-\lambda_{\varphi}\right) \sin 2 \delta}{1+\Lambda}, \\
& \text { with } \Lambda=\frac{1}{2}\left(\lambda_{\vartheta}-\lambda_{\varphi}\right) \sin ^{2} \delta-\frac{1}{2} \lambda_{\vartheta \varphi} \sin 2 \delta .
\end{aligned}
$$

The following frame-independent inequalities can be deduced by imposing rotation-invariance properties (for example, $\lambda_{\vartheta}$ never exceeds 1 in any frame) and using (9) and (11):

$\left|\lambda_{\varphi}\right| \leq \frac{1}{2}\left(1+\lambda_{\vartheta}\right), \quad\left|\lambda_{\vartheta \varphi}\right| \leq \frac{1}{2}\left(1-\lambda_{\varphi}\right)$

and, for $\lambda_{\varphi} \leq-1 / 3, \quad\left(2 \lambda_{\varphi}+1\right)^{2}+2 \lambda_{\vartheta \varphi}^{2} \leq 1$.

These relations imply the bounds $\left|\lambda_{\varphi}\right| \leq 1$ and $\left|\lambda_{\vartheta \varphi}\right| \leq$ $\sqrt{2} / 2$. More interestingly, we can see that $\left|\lambda_{\varphi}\right| \leq 0.5$ when $\lambda_{\vartheta}=0$ and must vanish when $\lambda_{\vartheta} \rightarrow-1$. The most general phase space for the three angular parameters is represented in Fig. 6. There is an alternative notation, widespread in the literature, where the coefficients $\lambda, v / 2$ and $\mu$ replace, respectively, $\lambda_{\vartheta}, \lambda_{\varphi}$ and $\lambda_{\vartheta \varphi}$. In that case, hence, we have $|v| \leq 2$

To illustrate the importance of the choice of the observation frame, we consider specific examples assuming, for simplicity, that the observation axis is perpendicular to the natural axis $\left(\delta= \pm 90^{\circ}\right)$. This case is of physical relevance since when the decaying particle is produced with small longitudinal momentum $\left(\left|p_{\mathrm{L}}\right| \ll p_{\mathrm{T}}\right.$, a frequent kinematic configuration in collider experiments) the CS and HX frames are actually perpendicular to one another. When $\delta=90^{\circ}$, a natural "transverse" polarization $\left(\lambda_{\vartheta}=+1\right.$ and $\lambda_{\varphi}=\lambda_{\vartheta \varphi}=0$ ), for example, transforms (21) into an observed polarization of opposite sign (but not fully "longitudinal"), $\lambda_{\vartheta}^{\prime}=-1 / 3$, with a significant azimuthal anisotropy, $\lambda_{\varphi}^{\prime}=1 / 3$, shown in Fig. 5(c). In terms of angular momentum wave functions, a state which is fully "transverse" with respect to one quantization axis is a coherent superposition of $50 \%$ "transverse" and $50 \%$ "longitudinal" components 
Fig. 6 Allowed regions for the decay angular parameters

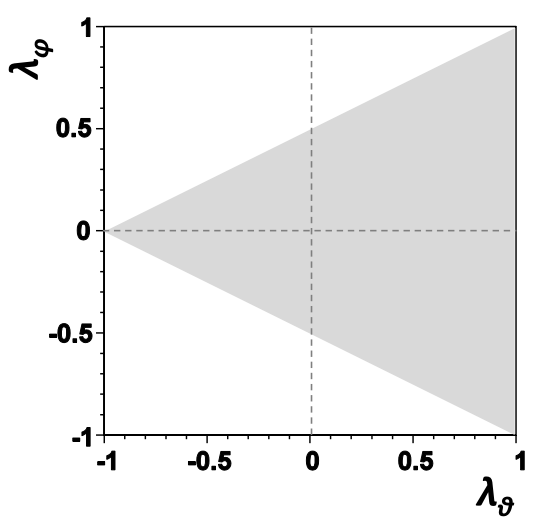

with respect to an axis rotated by $90^{\circ}(2)$ :

$|1, \pm 1\rangle \stackrel{90^{\circ}}{\longrightarrow} \frac{1}{2}|1,+1\rangle+\frac{1}{2}|1,-1\rangle \mp \frac{1}{\sqrt{2}}|1,0\rangle$.

The amplitude of the transition of this mixed state to the "rotated" dilepton state in (5) contains three terms with relative phases (due to the $\varphi$ dependence of the rotation matrix) giving rise to the observable azimuthal dependence. The same polar anisotropy $\lambda_{\vartheta}^{\prime}=-1 / 3$ would be measured in the presence of a mixture of at least two different processes resulting in 50\% "transverse" and 50\% "longitudinal" natural polarization along the chosen axis. In this case, however, no azimuthal anisotropy would be observed. As a second example, we note that a fully "longitudinal" natural polarization $\left(\lambda_{\vartheta}=-1\right)$ translates, in a frame rotated by $90^{\circ}$ with respect to the natural one, Fig. 5(d), into a fully "transverse" polarization $\left(\lambda_{\vartheta}^{\prime}=+1\right)$, accompanied by a maximal azimuthal anisotropy $\left(\lambda_{\varphi}^{\prime}=-1\right)$. In terms of angular momentum, the measurement in the rotated frame is performed on a coherent admixture of states,

$|1,0\rangle \stackrel{90^{\circ}}{\longrightarrow} \frac{1}{\sqrt{2}}|1,+1\rangle-\frac{1}{\sqrt{2}}|1,-1\rangle$,

while a natural "transverse" polarization would originate from the statistical superposition of uncorrelated $|1,+1\rangle$ and $|1,-1\rangle$ states. The two physically very different cases of a natural transverse polarization observed in the natural frame, shown in Fig. 5(a), and a natural longitudinal polarization observed in a rotated frame, shown in Fig. 5(d), are experimentally indistinguishable when the azimuthal anisotropy parameter is integrated out. These examples show that a measurement (or theoretical calculation) consisting only in the determination of the polar parameter $\lambda_{\vartheta}$ in one frame contains an ambiguity which prevents fundamental (model-independent) interpretations of the results. The polarization is only fully determined when both the polar and the azimuthal components of the decay distribution are known, or when the distribution is analyzed in at least two geometrically complementary frames.

\section{Effect of production kinematics on the observed decay kinematics}

Ideally, the dependence of the polarization on the momentum components of the produced quarkonium should reflect the relative contribution of individual production processes in different kinematic regimes, thereby providing information of fundamental physical interest. However, the observations are, in general, affected by some experimental limitations, which must be carefully taken in consideration. First, the frame-dependent polarization parameters $\lambda_{\vartheta}, \lambda_{\varphi}$ and $\lambda_{\vartheta \varphi}$ can be affected by a strong explicit kinematic dependence (encoded in the parameter $\delta$ in (21)), reflecting the change in direction of the chosen experimental axis (with respect to the "natural axis") as a function of the quarkonium momentum. Second, detector acceptances and event samples with limited statistics induce a dependence of the measurement on the distribution of events effectively accepted by the experimental apparatus.

To better explain the first problem, let us consider the HX and CS frames as the experimental and natural frames, respectively. We start by calculating the angle between the polarization axes of the CS and HX frames as a function of the quarkonium momentum. The beam momenta in the "laboratory" frame (centre of mass of the colliding particles), written in longitudinal and transverse components with respect to the quarkonium direction, are $\boldsymbol{P}_{1}=-\boldsymbol{P}_{2}=P \cos \Theta \hat{\imath}_{\|}+$ $P \sin \Theta \hat{\imath}_{\perp}$, where $P$ is their modulus and $\Theta$ is the angle formed by the quarkonium momentum with respect to the beam axis, defined in terms of the quarkonium momentum $\boldsymbol{p}$ as $\cos \Theta=p_{\mathrm{L}} / p$. When boosted to the quarkonium rest frame, the two vectors become (neglecting the masses of the colliding particles) $\boldsymbol{P}_{1}^{\prime}=(\gamma P \cos \Theta-\beta \gamma P) \hat{\imath}_{\|}+P \sin \Theta \hat{\imath}_{\perp}$ and $\boldsymbol{P}_{2}^{\prime}=(-\gamma P \cos \Theta-\beta \gamma P) \hat{\imath}_{\|}-P \sin \Theta \hat{\imath}_{\perp}$, where $\gamma=$ $E / m$ is the Lorentz factor of the quarkonium state, and $\beta=p / E=\sqrt{1-1 / \gamma^{2}}$. The unit vectors indicating the $z$ 
axis directions in the $\mathrm{HX}$ and $\mathrm{CS}$ frames are

$$
\begin{aligned}
& \hat{z}_{\mathrm{HX}}=-\frac{\boldsymbol{P}_{1}^{\prime}+\boldsymbol{P}_{2}^{\prime}}{\left|\boldsymbol{P}_{1}^{\prime}+\boldsymbol{P}_{2}^{\prime}\right|}=\frac{\boldsymbol{p}}{p}, \\
& \hat{z}_{\mathrm{CS}}=\frac{P_{2}^{\prime} \boldsymbol{P}_{1}^{\prime}-P_{1}^{\prime} \boldsymbol{P}_{2}^{\prime}}{\left|P_{2}^{\prime} \boldsymbol{P}_{1}^{\prime}-P_{1}^{\prime} \boldsymbol{P}_{2}^{\prime}\right|} .
\end{aligned}
$$

By definition, $\hat{z}_{\mathrm{HX}}=\hat{\imath}_{\|}$, while $\hat{z}_{\mathrm{CS}}$ can now be expressed as $\cos \tau \hat{\imath}_{\|}+\sin \tau \hat{\imath}_{\perp}, \tau$ being the angle between the two axes:

$$
\begin{aligned}
& \cos \tau=\frac{\frac{1}{\gamma} \cos \Theta}{\sqrt{\frac{1}{\gamma^{2}} \cos ^{2} \Theta+\sin ^{2} \Theta}}=\frac{m p_{\mathrm{L}}}{m_{\mathrm{T}} p}, \\
& \sin \tau=\frac{\sin \Theta}{\sqrt{\frac{1}{\gamma^{2}} \cos ^{2} \Theta+\sin ^{2} \Theta}}=\frac{E p_{\mathrm{T}}}{m_{\mathrm{T}} p} .
\end{aligned}
$$

We see that the result depends only on the momentum and mass of the quarkonium state $\left(m_{\mathrm{T}}=\sqrt{m^{2}+p_{\mathrm{T}}^{2}}\right)$. The angle $\delta$ entering in (21), equal to $\tau$ in magnitude, defines the positive rotation (respecting the right-hand rule) from one frame to the other. Its sign depends, therefore, on the exact conventions used for the orientation of the axes $y$ and $z$ of the polarization frames. In the convention where the $y$ axis is defined as

$\hat{y}=\frac{\left(\boldsymbol{P}_{1}^{\prime} \times \boldsymbol{P}_{2}^{\prime}\right)}{\left|\boldsymbol{P}_{1}^{\prime} \times \boldsymbol{P}_{2}^{\prime}\right|}$

and the $z$ axis is defined by (25), with the "first" beam oriented as the laboratory $z$ axis, the positive rotation is the one bringing the $\mathrm{HX}$ axis to coincide with the $\mathrm{CS}$ axis. We thus write, using (26),

$\delta_{\mathrm{HX} \rightarrow \mathrm{CS}}=-\delta_{\mathrm{CS} \rightarrow \mathrm{HX}}=\arccos \left(\frac{m p_{\mathrm{L}}}{m_{\mathrm{T}} p}\right)$.

Equation (21), containing terms of the form

$$
\begin{aligned}
& \sin ^{2} \delta_{\mathrm{HX} \rightarrow \mathrm{CS}}=\sin ^{2} \delta_{\mathrm{CS} \rightarrow \mathrm{HX}}=\frac{p_{\mathrm{T}}^{2} E^{2}}{p^{2} m_{\mathrm{T}}^{2}}, \\
& \sin 2 \delta_{\mathrm{HX} \rightarrow \mathrm{CS}}=-\sin 2 \delta_{\mathrm{CS} \rightarrow \mathrm{HX}}=\frac{2 m p_{\mathrm{T}} p_{\mathrm{L}} E}{p^{2} m_{\mathrm{T}}^{2}},
\end{aligned}
$$

is now explicitly seen as a kinematic-dependent transformation.

As an example, we show in Fig. 7 how natural $\mathrm{J} / \psi$ polarizations $\lambda_{\vartheta}=+1$ and -1 in the CS frame (with $\lambda_{\varphi}=$ $\lambda_{\vartheta \varphi}=0$ and no intrinsic kinematic dependence) translate into different $p_{\mathrm{T}}$-dependent polarizations measured in the HX frame in different rapidity acceptance windows, representative of the acceptance ranges of several Tevatron and LHC experiments. Corresponding figures for the $\Upsilon(1 S)$ case can be seen in [27]. The same results, except for a change in the sign of $\lambda_{\vartheta \varphi}$, the only parameter depending on the sign of the rotation angle, are obtained if the roles of the two frames are inter-exchanged.

The change of sign of the rapidity does not change the $\lambda_{\vartheta}$ and $\lambda_{\varphi}$ curves. However, the sign of $\lambda_{\vartheta \varphi}$ can change from positive to negative rapidity, depending on the convention used for the orientation of the axes. If the axes are defined as in (25) and (27) at both positive and negative rapidity, always taking as "first" beam the one positively oriented in the laboratory, $\lambda_{\vartheta \varphi}$ (proportional to $\sin 2 \delta$ with $0<\delta<\pi$ ) is forced to change sign when the rapidity changes sign. Any measurement integrating events over a range in rapidity where the acceptance is symmetrical around zero would, therefore, yield $\lambda_{\vartheta \varphi}=0$. In order to avoid this cancellation, the axis definitions in (25) and (27) can be improved by inverting the orientations of $\hat{y}$ and $\hat{z}_{\mathrm{CS}}$ for negative rapidity (correspondingly restricting the domain of the rotation angle to $0<\delta<\pi / 2$ ).

Having seen how the strong kinematic dependence induced by the choice of the observation frame can mimic and/or mask the fundamental ("intrinsic") dependencies reflecting the production mechanisms, let us now discuss the additional problems caused by common experimental limitations. Experiments can only measure the net polarization of the specific cocktail of quarkonium events accepted by the detector, trigger and analysis cuts. Moreover, each measured value necessarily implies an integration over certain ranges (bins or cells) of the quarkonium momentum components. If the polarization depends on the kinematics, this binning effectively biases the measured angular parameters, in different ways for experiments having different differential acceptances. In other words, two experiments covering the same kinematic interval can measure different average polarizations. This problem can be solved by presenting the results in narrow intervals of the probed phase space. Similarly, theoretical calculations aimed at comparisons with experimental data should consider how the momentum distributions are distorted by the acceptances of those experiments. Alternatively, the predictions should avoid kinematic integrations or, even better, be provided as event-level information to be embedded in the Monte Carlo simulations of the experiments. These considerations provide a further motivation for reporting measurements and theoretical calculations in frame-independent terms, as we will discuss in the next section.

\section{A frame-invariant approach}

The general frame-transformation relations in (21) imply the existence of an invariant quantity, definable in terms of $\lambda_{\vartheta}$, $\lambda_{\varphi}$ and $\lambda_{\vartheta \varphi}$, in one of the following equivalent forms:

$\mathcal{F}_{\left\{c_{1}, c_{2}, c_{3}\right\}}=\frac{\left(3+\lambda_{\vartheta}\right)+c_{1}\left(1-\lambda_{\varphi}\right)}{c_{2}\left(3+\lambda_{\vartheta}\right)+c_{3}\left(1-\lambda_{\varphi}\right)}$, 
Fig. 7 Kinematic dependence of the $\mathrm{J} / \psi$ decay angular distribution seen in the HX frame, for natural polarizations $\lambda_{\vartheta}=+1(\mathbf{a}-\mathbf{b}-\mathbf{c})$ and $\lambda_{\vartheta}=-1$ (d-e-f) in the CS frame. The curves correspond to different rapidity intervals; from the solid line: $|y|<0.6(\mathrm{CDF}),|y|<0.9$ (ALICE), $|y|<1.8$ (D0), $|y|<2.5$ (ATLAS and CMS), $2<y<5$ (LHCb). For simplicity the event populations were generated flat in rapidity. The sign of $\lambda_{\vartheta \varphi}$ depends on the definition of the $y$ axis of the polarization frame, here taken as $\operatorname{sign}\left(p_{\mathrm{L}}\right)\left(\boldsymbol{P}_{1}^{\prime} \times \boldsymbol{P}_{2}^{\prime}\right) /\left|\boldsymbol{P}_{1}^{\prime} \times \boldsymbol{P}_{2}^{\prime}\right|$, where $\boldsymbol{P}_{1,2}^{\prime}$ are the momenta of the colliding particles in the meson's rest frame


where $c_{1}, c_{2}$ and $c_{3}$ are real numbers. An account of the fundamental meaning of the frame-invariance of these quantities can be found in [28]. We will consider here, specifically, the form

$\tilde{\lambda} \equiv \mathcal{F}_{\{-3,0,1\}}=\frac{\lambda_{\vartheta}+3 \lambda_{\varphi}}{1-\lambda_{\varphi}}$.

In the special case when the observed distribution is the superposition of $n$ "elementary" distributions of the kind $1+\lambda_{\vartheta}^{(i)} \cos ^{2} \vartheta$, with event weights $f^{(i)}$, with respect to $n$ different polarization axes, $\tilde{\lambda}$ represents a weighted average of the $n$ polarizations, insensitive to the orientations of the corresponding axes:

$\tilde{\lambda}=\sum_{i=1}^{n} \frac{f^{(i)}}{3+\lambda_{\vartheta}^{(i)}} \lambda_{\vartheta}^{(i)} / \sum_{i=1}^{n} \frac{f^{(i)}}{3+\lambda_{\vartheta}^{(i)}}$.

The determination of an invariant quantity is immune to "extrinsic" kinematic dependencies induced by the observa- tion perspective and is, therefore, less acceptance-dependent than the determination of the standard anisotropy parameters $\lambda_{\vartheta}, \lambda_{\varphi}$ and $\lambda_{\vartheta \varphi}$.

This is shown in Fig. 8, where we consider, for illustration, that $60 \%$ of the $\mathrm{J} / \psi$ events have natural polarization $\lambda_{\vartheta}=+1$ in the CS frame while the remaining fraction has $\lambda_{\vartheta}=+1$ in the HX frame. Although the polarizations of the two event subsamples are intrinsically independent of the production kinematics, in neither frame, CS or HX, will measurements performed in different transverse and longitudinal momenta windows find identical results. However, in this case as well as in the simpler case of Fig. 7(ab-c), any arbitrary choice of the experimental observation frame will always yield the value $\tilde{\lambda}=+1$, independently of kinematics. This particular case, where all contributing processes are transversely polarized, is formally equivalent to the Lam-Tung relation [16], as discussed in [28]. Analogously, the example represented in Fig. 7(d-e-f), or any 
Fig. 8 Kinematic dependence of the $\mathrm{J} / \psi$ decay angular distribution seen in the HX $(\mathbf{a}-\mathbf{b}-\mathbf{c})$ and CS $(\mathbf{d}-\mathbf{e}-\mathbf{f})$ frames, when $60 \%$ (40\%) of the events have full transverse polarization in the CS (HX) frame. The curves represent measurements in different acceptance ranges, as detailed in Fig. 7.

Corresponding figures for the $\Upsilon(1 S)$ case can be seen in [27]
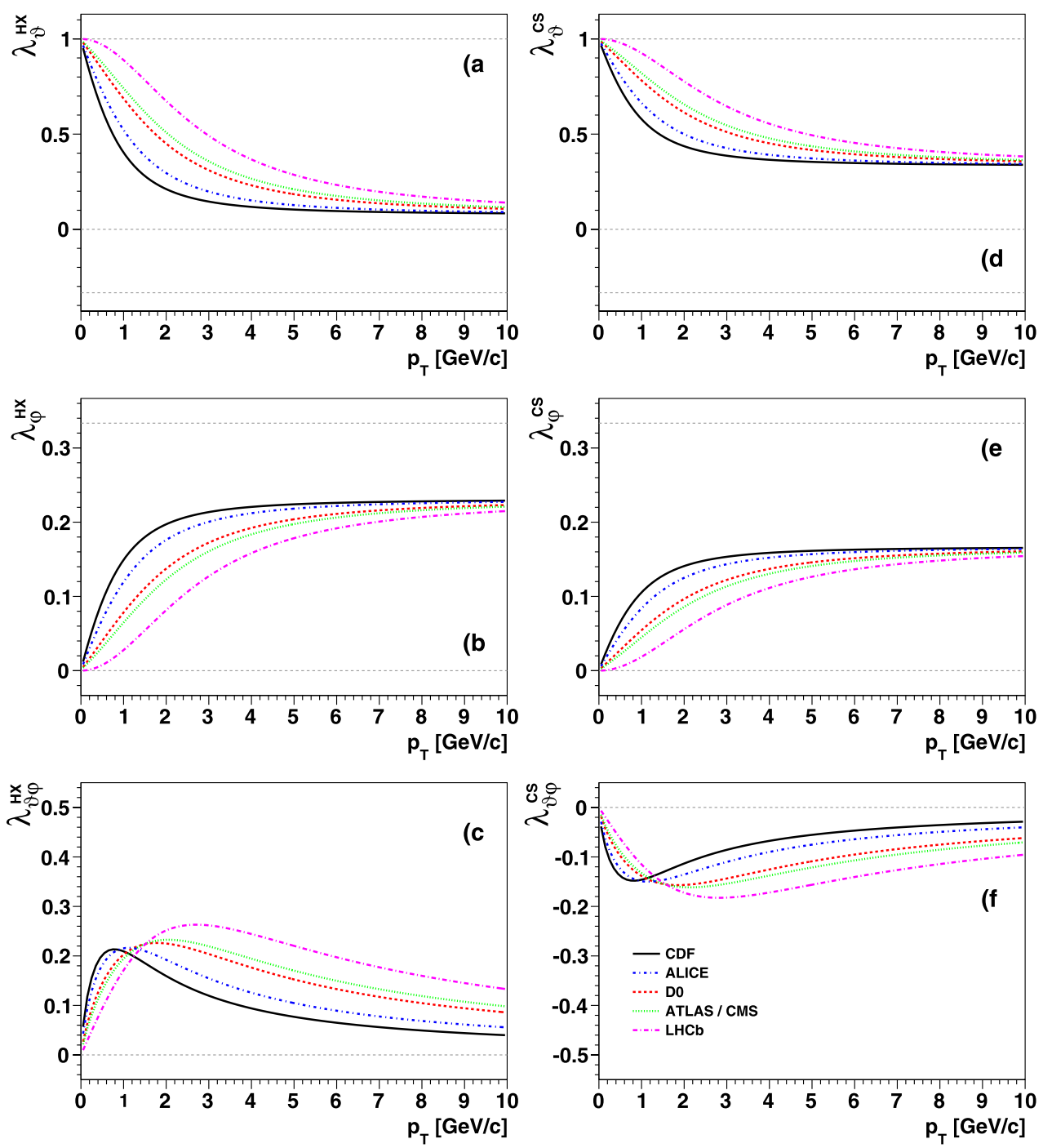

other case where all polarizations are longitudinal, yields $\tilde{\lambda}=-1$.

The existence of frame-invariant parameters also provides a useful instrument for experimental analyses. Checking, for example, that the same value of an invariant quantity (30) is obtained, within systematic uncertainties, in two distinct polarization frames is a non-trivial verification of the absence of unaccounted systematic effects. In fact, detector geometry and/or data selection constraints may strongly polarize the reconstructed dilepton events. Background processes also affect the measured polarization, if not well subtracted. The spurious anisotropies induced by detector effects and background do not obey the frame transformation rules characteristic of a physical $J=1$ state. If not well corrected and subtracted, these effects will distort the shape of the measured decay distribution differently in different polarization frames. In particular, they will violate the frame-independent relations between the angular parameters. Any two physical polarization axes (defined in the rest frame of the meson and belonging to the production plane) may be chosen to perform these "sanity tests". The HX and CS axes are ideal choices at high $p_{\mathrm{T}}$ and midrapidity, where they tend to be orthogonal to each other (in (29), $\sin ^{2} \delta \rightarrow 1$ for $p_{\mathrm{T}} \gg m$ and/or $\left.p_{\mathrm{T}} \gg\left|p_{\mathrm{L}}\right|\right)$. At forward rapidity and low $p_{\mathrm{T}}$, we can maximize the significance of the test by using the CS axis and the "perpendicular helicity axis" [29], which coincides with the helicity axis at zero rapidity and remains orthogonal to the CS axis at nonzero rapidity. Given that $\tilde{\lambda}$ is "homogeneous" to the anisotropy parameters, the difference $\tilde{\lambda}^{(\mathrm{B})}-\tilde{\lambda}^{(\mathrm{A})}$ between the results obtained in two frames provides a direct evaluation of the level of systematic errors not accounted in the analysis. 


\section{Effect of intrinsic parton transverse momentum}

In this section we describe how the geometry of the CS frame is related to the kinematics of the production process. It can be recognized from (26) that the vector $\hat{z}_{\mathrm{CS}}$ indicates the direction of the laboratory $z$ axis (that is, the beam line) as seen in the quarkonium rest frame. In this frame, any length will be Lorentz contracted by a factor $1 / \gamma$ along the quarkonium boost direction, but not along the transverse directions. In the quarkonium rest frame (as well as in the laboratory) the direction of the beam line coincides with the direction of the relative motion of the colliding partons ("parton axis"), when their transverse momenta are neglected (and exactly when averaging a large sample of events). This approximation affects the experimental determination of an angular distribution naturally of the kind $1+\lambda_{\vartheta}^{*} \cos ^{2} \vartheta^{*}$ with respect to the parton axis $z^{*}$. In the following considerations we fix a coordinate system having the $z$ axis along the dilepton direction in the laboratory and the $x z$ plane coinciding with the production plane. We then define the directions of the beam axis and of the parton axis in the laboratory as, respectively,

$\hat{B}=(\sin \Theta, 0, \cos \Theta)$

$\hat{B}^{\prime}=\left(\sin \Theta^{\prime} \cos \Phi^{\prime}, \sin \Theta^{\prime} \sin \Phi^{\prime}, \cos \Theta^{\prime}\right)$,

where $\Theta$ and $\Theta^{\prime}$ are the angles they form with respect to the dilepton direction. The presence of the angle $\Phi^{\prime}$ denotes the fact that, due to the intrinsic transverse momenta of the partons, the vector $\hat{B}^{\prime}$ does not belong, in general, to the production plane. The angle $\Delta$ between the two directions in the laboratory is given by

$\cos \Delta=\sin \Theta \sin \Theta^{\prime} \cos \Phi^{\prime}+\cos \Theta \cos \Theta^{\prime}$.

When boosted to the dilepton rest frame, the two vectors become

$\hat{b}=\frac{\left(\sin \Theta, 0, \frac{1}{\gamma} \cos \Theta\right)}{\sqrt{\sin ^{2} \Theta+\frac{1}{\gamma^{2}} \cos ^{2} \Theta}}$,

$\hat{b}^{\prime}=\frac{\left(\sin \Theta^{\prime} \cos \Phi^{\prime}, \sin \Theta^{\prime} \sin \Phi^{\prime}, \frac{1}{\gamma} \cos \Theta^{\prime}\right)}{\sqrt{\sin ^{2} \Theta^{\prime}+\frac{1}{\gamma^{2}} \cos ^{2} \Theta^{\prime}}}$

and the cosine of the angle between them

$\cos \zeta=\frac{\cos \Delta-\beta^{2} \cos \Theta \cos \Theta^{\prime}}{\sqrt{1-\beta^{2} \cos ^{2} \Theta} \sqrt{1-\beta^{2} \cos ^{2} \Theta^{\prime}}}$.

The rotation by $\zeta$ from the parton axis to the beam line axis transforms the polarization parameter $\lambda_{\vartheta}$ according to the following expressions:

$\lambda_{\vartheta}^{\mathrm{CS}} \simeq\left(1-\frac{3+\lambda_{\vartheta}^{*}}{2}\left\langle\sin ^{2} \zeta\right\rangle\right) \lambda_{\vartheta}^{*}$,

$\lambda_{\varphi}^{\mathrm{CS}} \simeq \lambda_{\vartheta \varphi}^{\mathrm{CS}} \simeq 0$

These transformations do not represent a simple rotation as (21). Indeed, the magnitude of the polar anisotropy decreases, while no significant azimuthal anisotropy arises. In fact, the rotation plane (formed by the parton and beam lines) does not coincide with the experimentally defined production plane. The angle between the two planes changes from one event to the next, so that the azimuthal anisotropy deriving from the tilt between the "natural" polarization axis and the experimental axis tends to be smeared out in the integration over all events. Since $\cos \Delta \simeq 1-\frac{1}{2} \sin ^{2} \Delta$ and (approximately event-by-event, and exactly on average) $\cos \Theta^{\prime} \simeq \cos \Theta$, from (36) we obtain

$\left\langle\sin ^{2} \zeta\right\rangle \simeq \frac{\left\langle\sin ^{2} \Delta\right\rangle}{1-\beta^{2} \cos ^{2} \Theta}=\frac{E^{2}}{m_{\mathrm{T}}^{2}}\left\langle\sin ^{2} \Delta\right\rangle$.

Denoting by $\boldsymbol{k}_{1,2}, \boldsymbol{k}_{1,2 \mathrm{~T}}$ and $E_{1,2}$ the total momenta, transverse momenta and energies of the two partons in the laboratory, the laboratory angle $\Delta$ satisfies

$\sin ^{2} \Delta=\frac{\left(\boldsymbol{k}_{1 \mathrm{~T}}-\boldsymbol{k}_{2 \mathrm{~T}}\right)^{2}}{\left(\boldsymbol{k}_{1}-\boldsymbol{k}_{2}\right)^{2}} \simeq \frac{\left(\boldsymbol{k}_{1 \mathrm{~T}}-\boldsymbol{k}_{2 \mathrm{~T}}\right)^{2}}{\left(E_{1}+E_{2}\right)^{2}}$

and, on average,

$\left\langle\sin ^{2} \Delta\right\rangle \simeq \frac{2\left\langle\boldsymbol{k}_{\mathrm{T}}^{2}\right\rangle}{\left(E_{1}+E_{2}\right)^{2}}$,

where we have defined the average parton squared transverse momentum as $\left\langle\boldsymbol{k}_{\mathrm{T}}^{2}\right\rangle=\left(\left\langle\boldsymbol{k}_{1 \mathrm{~T}}^{2}\right\rangle+\left\langle\boldsymbol{k}_{2 \mathrm{~T}}^{2}\right\rangle\right) / 2$.

Considering now the specific case of Drell-Yan production at low $p_{\mathrm{T}}$, we can assume an approximate equality between total parton energy and dilepton energy, $E_{1}+E_{2} \simeq E$, and, moreover, $\left\langle m_{\mathrm{T}}^{2}\right\rangle \simeq m^{2}+2\left\langle\boldsymbol{k}_{\mathrm{T}}^{2}\right\rangle$. Combining (37) (with $\left.\lambda_{\vartheta}^{*}=+1\right)$, (38) and (40), we find that the measurement of the polarization of low- $p_{\mathrm{T}}$ Drell-Yan dileptons provides an estimate of the "effective" parton transverse momentum:

$\left\langle\boldsymbol{k}_{\mathrm{T}}^{2}\right\rangle \simeq \frac{m^{2}}{2} \frac{1-\lambda_{\vartheta}^{\mathrm{CS}}}{1+\lambda_{\vartheta}^{\mathrm{CS}}}$

The average Drell-Yan polarization $\lambda_{\vartheta}^{\mathrm{CS}}=1.008 \pm 0.026$ measured by E866 [14], in proton-copper collisions for $\langle m\rangle \simeq 10 \mathrm{GeV} / c^{2}$ and $p_{\mathrm{T}}<4 \mathrm{GeV} / c$, implies, therefore, that

$\left\langle\boldsymbol{k}_{\mathrm{T}}^{2}\right\rangle<0.5 \mathrm{GeV}^{2} / c^{2}$ at $68 \%$ C.L. and

$\left\langle\boldsymbol{k}_{\mathrm{T}}^{2}\right\rangle<1.0 \mathrm{GeV}^{2} / c^{2}$ at $95 \%$ C.L. 
Tighter limits could be derived from precise low-mass measurements, given that the polarization smearing effects are essentially proportional to $\mathrm{m}^{-2}$. Unfortunately, the existing (pion-induced) measurements [30, 31], though very precise and extending down to $4 \mathrm{GeV} / c^{2}$, present large azimuthal anisotropies of dubious interpretation and are scarcely suitable for this purpose.

We can now estimate the maximum magnitude of the smearing effects that can be foreseen for the observable quarkonium polarization when the natural polarization axis is the parton axis. Combining again (37), (38) and (40), this time with $E_{1}+E_{2} \geq E$, we find that the magnitude of the polarization is reduced by the fraction

$\left|\frac{\lambda_{\vartheta}^{\mathrm{CS}}-\lambda_{\vartheta}^{*}}{\lambda_{\vartheta}^{*}}\right| \lesssim \frac{\left(3+\lambda_{\vartheta}^{*}\right)\left\langle\boldsymbol{k}_{\mathrm{T}}^{2}\right\rangle}{m^{2}+p_{\mathrm{T}}^{2}}$.

For example, it cannot be excluded, considering the limit in (42), that a fully transverse natural polarization of the $\mathrm{J} / \psi$ along the parton axis is reduced by as much as $30 \%$, for $p_{\mathrm{T}}=2 \mathrm{GeV} / c$, when observed in the CS frame. This smearing effect should be one order of magnitude smaller for $\mathrm{J} / \psi$ mesons of $p_{\mathrm{T}}=10 \mathrm{GeV} / c$. On the other hand, given the strong dependence of the effect on the dilepton mass, bottomonium polarization measurements are practically insensitive to the parton transverse momentum even at low $p_{\mathrm{T}}$. This prediction is consistent with the already quoted E866 results, showing $\Upsilon(2 S+3 S)$ polarizations in the CS frame always compatible with +1 , within a $\sim 15 \%$ uncertainty, in four $p_{\mathrm{T}}$ bins between 0 and $4 \mathrm{GeV} / c$.

\section{A few concrete examples}

We conclude with some examples of measurements illustrating concepts described in the previous sections.

We have already referred to the E866 measurement of a full transverse $\Upsilon(2 S+3 S)$ polarization in the CS frame. The result is represented in Fig. 9(a), as a function of $p_{\mathrm{T}}$. A similarly constant behaviour, consistent with a Drell-Yanlike polarization, has been measured by this experiment as a function of $x_{\mathrm{F}}$, in the range [0,0.5], confirming that the adoption of the CS frame is, in this case, an optimal choice. It is true that, in special kinematic conditions, the transverse polarization observed in one frame could, in reality, be the reflection of a natural longitudinal polarization in another frame, as shown in Fig. 7(d). However, a maximal polarization independent of the production kinematics in the CS frame must directly reflect the spin configuration of the interacting partons (as is well known to be the case in DrellYan production, a paradigmatic example of natural transverse polarization in the CS frame).

To better illustrate the importance of an optimal choice of the reference frame, we will now consider what the E866 experiment would have measured, had the analysis been made with a different choice. As a reasonable approximation, we assume that the azimuthal distribution is exactly isotropic in the CS frame. The polar anisotropy that would be observed in the HX frame is shown in Fig. 9(b), where the curve includes an extrapolation to higher $p_{\mathrm{T}}$ assuming that in the CS frame the distribution continues to have the shape $1+\cos ^{2} \vartheta$. A measurement performed in the HX frame would show, quite misleadingly, a polarization changing from fully transverse to partially longitudinal. The strong signature of "natural" transverse polarization evidenced by the data in the CS frame would become unrecognizable, although it could, in principle, be reconstructed back if (and only if) the azimuthal anisotropy were also measured.

Seeing how the curve in Fig. 9(b) qualitatively resembles the pattern measured by $\mathrm{CDF}$ for the $\mathrm{J} / \psi$, it is natural to wonder how that measurement, made in the HX frame, would look like in the CS frame. Unfortunately, in this case the measurement itself, a slight longitudinal polarization, does not suggest any educated guess on what we could assume for the unmeasured azimuthal anisotropy. For example, as shown in Fig. 10, if the distribution in the HX frame were azimuthally isotropic, the measured polarization would correspond to a practically undetectable polarization in the CS frame (dashed line). However, if we take into account all physically possible values of the azimuthal anisotropy, as allowed by the triangular relation represented in Fig. 6, we can only derive a broad spectrum of possible CS polarizations, approximately included between -0.5 and +1 (shaded band). This example shows how a measurement re-
Fig. 9 The E866 measurement of the $\Upsilon(2 S+3 S)$ polarization in the CS (a) and a guess of how it would be observed in the HX frame, extrapolating to higher $p_{\mathrm{T}}(\mathbf{b})$
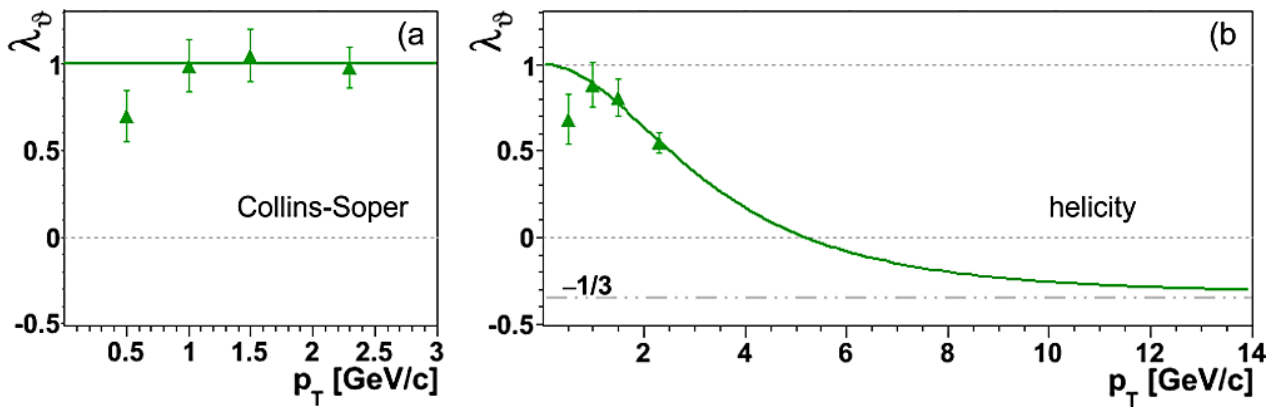


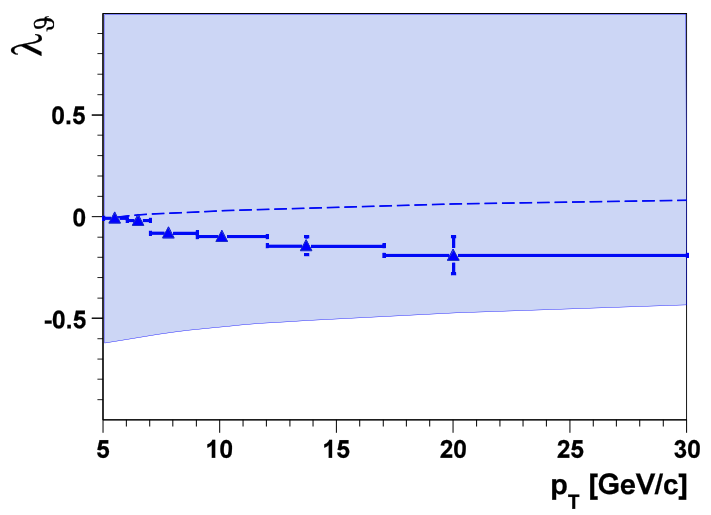

Fig. 10 The $\mathrm{CDF} J / \psi$ polarization measurement in the helicity frame (data points) and the range for the corresponding polarization in the CS, allowing for all possible values of the azimuthal anisotropy (shaded band). The dashed line is the CS polarization for $\lambda_{\varphi}^{\mathrm{HX}}=0$

porting only the polar anisotropy is amenable to several interpretations in fundamental terms, often corresponding to drastically different physical cases. One possible hypothesis would be that all processes are naturally polarized in the HX frame and that transverse and longitudinal polarizations are superimposed in proportions varying from approximately $2 / 3$ transverse and $1 / 3$ longitudinal at $p_{\mathrm{T}}=$ $5 \mathrm{GeV} / c\left(\lambda_{\vartheta} \simeq 0\right)$ to around $60 \% / 40 \%$ at $p_{\mathrm{T}}=20 \mathrm{GeV} / c$ $\left(\lambda_{\vartheta} \simeq-0.2\right)$. In this case, no azimuthal anisotropy should be observed in the HX frame. Alternatively, we can consider a scenario where the observed slightly longitudinal HX polarization is actually the result of a mixture of two processes, both producing $\mathrm{J} / \psi$ mesons with fully transverse polarizations, but one in the HX frame and the other in the CS frame. Figure 11 (top) shows that this scenario is perfectly compatible with the CDF $\lambda_{\vartheta}$ measurement if the proportion $f_{\mathrm{HX}} /\left(f_{\mathrm{HX}}+f_{\mathrm{CS}}\right)$ between the two sub-processes is assumed to vary linearly between $30 \%$ at $p_{\mathrm{T}}=5 \mathrm{GeV} / c$ and $15 \%$ at $p_{\mathrm{T}}=20 \mathrm{GeV} / c$. The difference with respect to the previous hypothesis is that now we would measure a significant azimuthal anisotropy, $\lambda_{\varphi} \simeq 0.3$, as shown in Fig. 11 (bottom). As an attempt to reconcile low- $p_{\mathrm{T}}$ measurements with collider data, [11] described one further conjecture, in which the polarization arises naturally in the CS frame, and becomes increasingly transverse with increasing total $\mathrm{J} / \psi$ momentum. Again, a direct measurement of $\lambda_{\varphi}$ (which, in this case, should be zero in the CS frame but positive and increasing in the HX frame) would easily clarify the situation.

We finish this section by illustrating the application of the frame-independent formalism as a tool to estimate residual systematic uncertainties in experimental data analyses. Figure 12 shows a putative set of $\mathrm{J} / \psi$ polarization measurements performed in the CS and HX frames, versus $p_{\mathrm{T}}$. While the $\lambda_{\vartheta}$ values seem to change significantly from one frame to the other, the two $\lambda_{\varphi}$ patterns are very similar. This
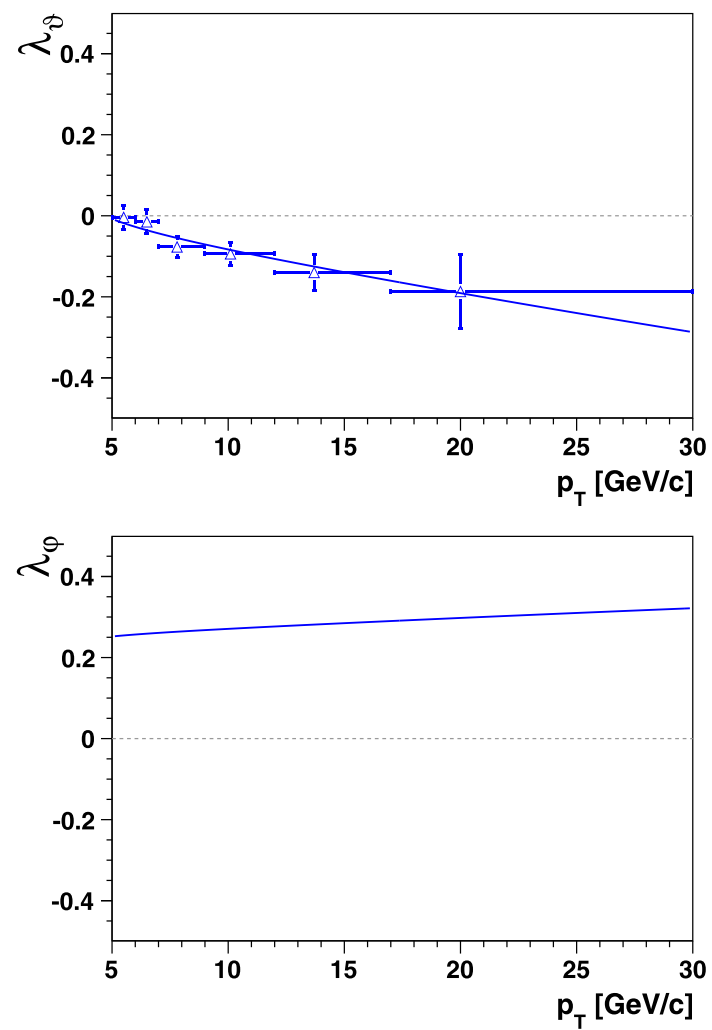

Fig. 11 The $p_{\mathrm{T}}$ dependence of the angular parameters $\lambda_{\vartheta}$ (top) and $\lambda_{\varphi}$ (bottom) as would be measured by CDF in the HX frame, according to a scenario where the $\mathrm{J} / \psi$ 's have always full transverse polarization, either in the CS frame or in the HX frame, with a suitable $p_{\mathrm{T}}$-dependent proportion between the two event samples. The data points represent the CDF measurement

observation should alert to a possible experimental artifact in the data analysis. We can evaluate the significance of the apparent contradiction by calculating the frame-invariant $\tilde{\lambda}$ variable in each of the two frames. For the case illustrated in Fig. 12, averaging the four represented $p_{\mathrm{T}}$ bins, we see that $\tilde{\lambda}$ in the HX frame is larger than in the CS frame by 0.5 (a rather large value, considering that the decay parameters are bound between -1 and +1 ). In other words, an experiment obtaining such measurements would learn from this simple exercise that its determination of the decay parameters must be biased by systematic errors of roughly this magnitude. Given the puzzles and contradictions existing in the published experimental results, as recalled in Sect. 1, the use of a frame-invariant approach to perform self-consistency checks, which can expose unaccounted systematic effects due to detector limitations and analysis biases, constitutes a non-trivial complementary aspect of the new approach proposed in this paper for quarkonium polarization measurements. 

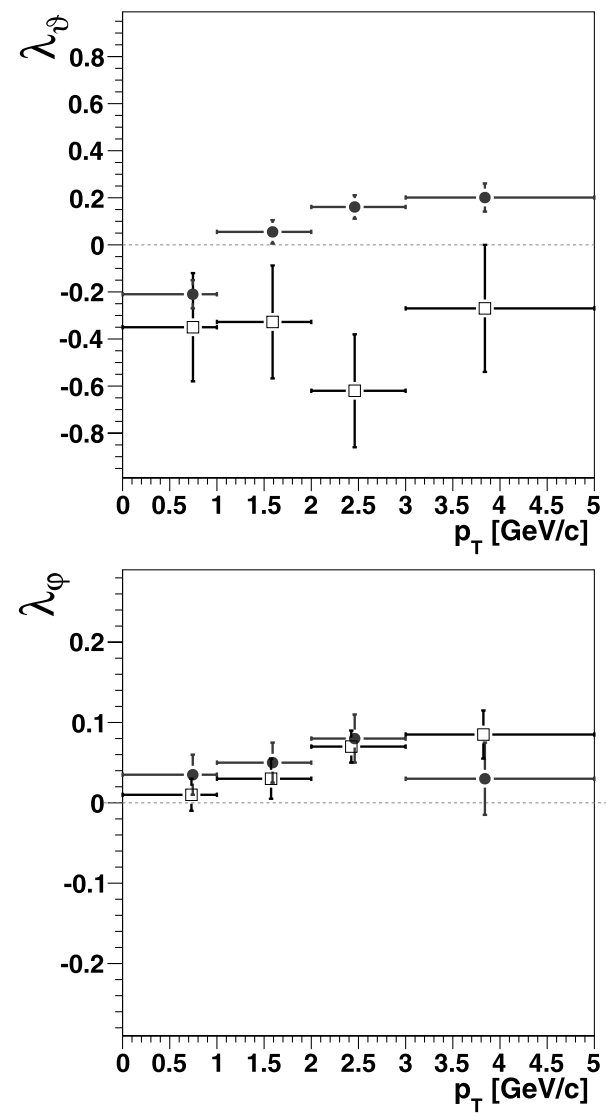

Fig. 12 Example of a "gedankenexperiment" where the $\mathrm{J} / \psi$ polarization measurements in the CS and HX frames (empty and filled symbols, respectively) would be inconsistent with each other

\section{Summary and conclusions}

Motivated by several puzzles affecting the existing measurements of quarkonium polarization, we present in this paper a set of proposals which should improve the experimental determination of the $\mathrm{J} / \psi$ and $\Upsilon$ polarizations. They are summarized in the next paragraphs.

Measurements and calculations of vector quarkonium polarization should provide results for the full dilepton decay angular distribution (a three-parameter function) and not only for the polar anisotropy parameter. Only in this way can the measurements and calculations represent unambiguous determinations of the average angular momentum composition of the produced quarkonium state in terms of the three base eigenstates, with $J_{z}=+1,0,-1$.

It is advisable to perform the experimental analyses in at least two different polarization frames. In fact, the selfevidence of certain signature polarization cases (e.g. a full polarization with respect to a specific axis) can be spoiled by an unfortunate choice of the reference frame, which can lead to artificial ("extrinsic") dependencies of the results on the kinematics and on the experimental acceptance.
The measured dependence of the polarization on the production kinematics is necessarily influenced by the differential experimental acceptance, i.e. by the kinematic distribution of the population of the accepted events. This problem, which is not solved by acceptance corrections, can be minimized by providing the results in narrow cells in quarkonium rapidity and transverse momentum. Theoretical calculations should be provided as event-level inputs to Monte Carlo generators which can be tailored to the specific performance capabilities of each experiment.

The decay angular distribution can be characterized by a frame-independent quantity, such as $\tilde{\lambda}$, calculable in terms of the polar and azimuthal anisotropy parameters. The existence of such frame-invariant quantities can be used during the data analysis phase to perform self-consistency checks that can expose previously unaccounted biases, caused, for instance, by the detector limitations or by the event selection criteria.

Besides providing a much needed control over systematic experimental biases, the variable $\tilde{\lambda}$ also provides relevant physical information: it characterizes the shape of the angular distribution, reflecting "intrinsic" spin-alignment properties of the decaying state, irrespectively of the specific geometrical framework chosen by the observer. For instance, we obtain $\tilde{\lambda}=+1$ for the shapes shown in Fig. 5(a) and (c), and $\tilde{\lambda}=-1$ for the shapes shown in the panels (b) and (d). A very important advantage of re-expressing the framedependent polar and azimuthal anisotropies in terms of a frame-invariant quantity is the exact cancellation of extrinsic dependencies on kinematics and acceptances, enabling more robust comparisons with other experiments and with theory. The calculation of $\tilde{\lambda}$ requires, anyhow, the determination of the full decay distribution in a chosen reference frame and, obviously, does not replace this standard procedure. Moreover, the three frame-dependent parameters $\left(\lambda_{\vartheta}\right.$, $\lambda_{\varphi}$ and $\lambda_{\vartheta \varphi}$ ) can provide information on the direction of the spin-alignment of the decaying particle (when this direction is univocally defined) and, therefore, on the topological properties of the dominant production mechanism. For instance, the measurement of a full transverse polarization in the CS frame represents a direct observation of the spin alignments of the interacting partons, as we know from Drell-Yan production. On the other hand, in the presence of a superposition of production processes with polarizations along different axes, measuring the frame-dependent anisotropies will not provide, in general, much information on the polarizations involved or on their natural alignment directions, while the value of $\tilde{\lambda}$ will immediately tell us if the processes involved have a predominantly transverse or longitudinal nature.

Stripped-down analyses which only measure the polar anisotropy in a single reference frame, as often done in past experiments, give more information about the frame 
selected by the analyst ("is the adopted quantization direction an optimal choice?") than about the physical properties of the produced quarkonium ("along which direction is the spin aligned, on average?"). For example, a natural longitudinal polarization will give any desired $\lambda_{\vartheta}$ value, from -1 to +1 , if observed from a suitably chosen reference frame. Lack of statistics is not a reason to "reduce the number of free parameters" if the resulting measurements become ambiguous. The forthcoming measurements of quarkonium polarization in proton-proton collisions at the LHC have the potential of providing a very important step forward in our understanding of quarkonium production, if the experiments adopt a more robust analysis framework, incorporating the ideas presented in this paper.

Acknowledgements P.F., J.S. and H.K.W. acknowledge support from Fundação para a Ciência e a Tecnologia, Portugal, under contracts SFRH/BPD/42343/2007, CERN/FP/109343/2009 and SFRH/BPD/ 42138/2007.

Open Access This article is distributed under the terms of the Creative Commons Attribution Noncommercial License which permits any noncommercial use, distribution, and reproduction in any medium, provided the original author(s) and source are credited.

\section{References}

1. N. Brambilla et al. (QWG Coll.), CERN Yellow Report 2005-005, hep-ph/0412158, and references therein

2. F. Abe et al. (CDF Coll.), Phys. Rev. Lett. 79, 572 (1997)

3. G.T. Bodwin, E. Braaten, G.P. Lepage, Phys. Rev. D 51, 1125 (1995)
4. G.T. Bodwin, E. Braaten, G.P. Lepage, Phys. Rev. D 55, 5853E (1997)

5. J.P. Lansberg, Eur. Phys. J. C 61, 693 (2009)

6. M. Beneke, M. Krämer, Phys. Rev. D 55, 5269 (1997)

7. A.K. Leibovich, Phys. Rev. D 56, 4412 (1997)

8. E. Braaten, B.A. Kniehl, J. Lee, Phys. Rev. D 62, 094005 (2000)

9. A. Abulencia et al. (CDF Coll.), Phys. Rev. Lett. 99, 132001 (2007)

10. P. Faccioli, C. Lourenço, J. Seixas, H.K. Wöhri, J. High Energy Phys. 10, 004 (2008)

11. P. Faccioli, C. Lourenço, J. Seixas, H.K. Wöhri, Phys. Rev. Lett. 102, 151802 (2009)

12. D. Acosta et al. (CDF Coll.), Phys. Rev. Lett. 88, 161802 (2002)

13. V.M. Abazov et al. (D0 Coll.), Phys. Rev. Lett. 101, 182004 (2008)

14. C.N. Brown et al. (E866 Coll.), Phys. Rev. Lett. 86, 2529 (2001)

15. T. Affolder et al. (CDF Coll.), Phys. Rev. Lett. 85, 2886 (2000)

16. C.S. Lam, W.K. Tung, Phys. Rev. D 18, 2447 (1978)

17. M. Artuso et al. (CLEO Coll.), Phys. Rev. D 80, 112003 (2009)

18. T.A. Armstrong et al. (E760 Coll.), Phys. Rev. D 48, 3037 (1993)

19. M. Ambrogiani et al. (E835 Coll.), Phys. Rev. D 65, 052002 (2002)

20. M. Ambrogiani et al. (E835 Coll.), Phys. Lett. B 610, 177 (2005)

21. E. Braaten, T.C. Yuan, Phys. Rev. Lett. 71, 1673 (1993)

22. H. Fritzsch, Phys. Lett. B 67, 217 (1977)

23. F. Halzen, Phys. Lett. B 69, 105 (1977)

24. K. Gottfried, J.D. Jackson, Nuovo Cimento 33, 309 (1964)

25. J.C. Collins, D.E. Soper, Phys. Rev. D 16, 2219 (1977)

26. D.M. Brink, G.R. Satchler, Angular Momentum, 3rd edn. (Clarendon Press, Oxford, 1993)

27. P. Faccioli, C. Lourenço, J. Seixas, Phys. Rev. D 81, 111502(R) (2010)

28. P. Faccioli, C. Lourenço, J. Seixas, Phys. Rev. Lett. 105, 061601 (2010)

29. E. Braaten, D. Kang, J. Lee, C. Yu, Phys. Rev. D 79, 014025 (2009)

30. S. Falciano et al. (NA10 Coll.), Z. Phys. C 31, 513 (1986)

31. J.S. Conway et al. (E615 Coll.), Phys. Rev. D 39, 92 (1989) 